\title{
Stability of Sinusoidal Responses of Marginally Stable Bandpass Sigma Delta Modulators
}

\author{
Charlotte Yuk-Fan Ho \\ Telephone: +44 (0)20 78827986 Fax: +44(0)20 78827997 Email: charlotte.ho@elec.qmul.ac.uk \\ Department of Electronic Engineering, Queen Mary, University of London, Mile End Road, London, E1 4NS, United Kingdom. \\ *Bingo Wing-Kuen Ling \\ Telephone: +44 (0)20 78482294 Fax: +44 (0)20 78482932 Email: wing-kuen.ling@kcl.ac.uk \\ Department of Electronic Engineering, Division of Engineering, King's College London, Strand, London, WC2R 2LS, United Kingdom. \\ Joshua D. Reiss \\ Telephone: +44 (0)20 78827982 Fax: +44 (0)20 78827997 Email: josh.reiss@elec.qmul.ac.uk \\ Department of Electronic Engineering, Queen Mary, University of London, Mile End Road, London, E1 4NS, United Kingdom.
}

\begin{abstract}
In this paper, we analyze the stability of the sinusoidal responses of second order interpolative marginally stable bandpass sigma delta modulators (SDMs) with the sum of the numerator and denominator polynomials equal to one and explore new results on the more general second order interpolative marginally stable bandpass SDMs. These results can be further extended to the high order interpolative marginally stable bandpass SDMs.
\end{abstract}

Index Terms-Interpolative marginally stable bandpass sigma delta modulators, resonance, sinusoidal responses.

\section{INTRODUCTION}

Since SDMs can perform analog-to-digital (A/D) conversion using simple, robust and inexpensive circuits, and can achieve very high signal-to-noise ratio (SNR) because of the noise shaping characteristics [1], SDMs are found in many industrial and consumer electronic products. One of the advantages of employing bandpass SDMs over the lowpass SDMs is to reduce the sampling frequency by operating the SDMs on high frequency narrowband signals, so bandpass SDMs become more popular and have been investigated in the communications, signal processing and circuits and systems societies extensively [2]-[5].

Since SDMs consist of a quantizer, which is a nonlinear component, in the feedback loop, the dynamical behaviors of SDMs could be very complex even though the loop filters in the SDMs are as simple as second order, rational, strictly proper and causal filters with a unit delay in the numerator [2]-[5]. It was found that the trajectory of second order bandpass SDMs may exhibit one or more ellipses or elliptic fractal patterns confined within two trapezoids when zero or step inputs are applied 
[2]-[5]. Although these results help for understanding the dynamical behaviors of second order bandpass SDMs, bandpass inputs should be employed for the analysis because bandpass SDMs shape away the noise from bandpass regions and operate at bandpass signals. Some simulation results based on bandpass inputs have been performed in [2]. In [6], statistical properties of the error signals have been investigated, but the behaviors of the SDMs have not been discussed. In [7], periodic behaviors of the output sequences have been discussed, but chaotic and divergent behaviors of the SDMs have not been explored.

In this paper, we start at studying the sinusoidal responses of the SDMs introduced in [2]-[5], then we explore new results on the more general second order interpolative marginally stable bandpass SDMs and extend these results to the high order interpolative marginally stable bandpass SDMs. The outline of this paper is as follows. The notations are introduced in Section II. Both the analytical and simulation results are presented in Section III. Finally, a conclusion is summarized in section IV.

\section{NOTATIONS}

Consider the second order interpolative marginally stable bandpass SDMs introduced in [2]-[5]. The transfer function of the loop filter of the SDMs is denoted as $F(z)=\frac{2 \cos \theta z^{-1}-z^{-2}}{1-2 \cos \theta z^{-1}+z^{-2}}$, where $\theta$ is the filter parameter depending on the sampling frequency and the operating frequency of the SDMs. For this second order marginally stable bandpass loop filter, $\theta$ is also the natural frequency of the loop filter and the SDMs shape away the noise from this frequency. Denote the input of the SDMs and the output of the loop filter are, respectively, $u(k)$ and $y(k)$. Then the SDM can be described by the following state space equation [2]-[5]:

$$
\begin{gathered}
\mathbf{x}(k+1)=\mathbf{A x}(k)+\mathbf{B}(\mathbf{u}(k)-\mathbf{s}(k)) \text { for } k \geq 0, \\
y(k)=\mathbf{C} \mathbf{x}(k)+\mathbf{D}(\mathbf{u}(k)-\mathbf{s}(k)) \text { for } k \geq 0,
\end{gathered}
$$

where $\mathbf{x}(k) \equiv\left[x_{1}(k) \quad x_{2}(k)\right]^{T} \equiv[y(k-2) \quad y(k-1)]^{T}$ is the state vector of the SDMs and the state variables are defined as the delay version of the output of the loop filter, $\mathbf{u}(k) \equiv[u(k-2) u(k-1)]^{T}$ is a vector containing the past two consecutive points from the input signal $u(k)$,

$$
\mathbf{A} \equiv\left[\begin{array}{cc}
0 & 1 \\
-1 & 2 \cos \theta
\end{array}\right]
$$

is the system matrix,

$$
\mathbf{B} \equiv\left[\begin{array}{cc}
0 & 0 \\
-1 & 2 \cos \theta
\end{array}\right]
$$

is the matrix associated with the input of the loop filter, $\mathbf{C}$ and $\mathbf{D}$ are the matrices associated with the output of the loop filter. Since the state variables are chosen as the delay version of the output of the loop filter, $\mathbf{C}$ and $\mathbf{D}$ are the last row of matrices $\mathbf{A}$ and $\mathbf{B}$, respectively, and

$$
\mathbf{s}(k) \equiv\left[Q\left(x_{1}(k)\right) \quad Q\left(x_{2}(k)\right)\right]^{T} \text { for } k \geq 0
$$


is the quantized state vector, in which the superscript ${ }^{T}$ denotes the transpose operator,

$$
Q(y) \equiv\left\{\begin{array}{cc}
1 & y \geq 0 \\
-1 & \text { otherwise }
\end{array}\right.
$$

is a one bit quantizer and $\theta \in(-\pi, \pi) \backslash\{0\}$. When $\theta \in\{-\pi, 0, \pi\}$, the system will become either lowpass or highpass SDMs, which are out of the scope of this paper because this paper only focuses on the bandpass SDMs. Since $\mathbf{s}(k)$ consists of only 4 possible values: $\left[\begin{array}{ll}1 & 1\end{array}\right]^{T},\left[\begin{array}{ll}1 & -1\end{array}\right]^{T},\left[\begin{array}{ll}-1 & -1\end{array}\right]^{T}$, and $\left[\begin{array}{ll}-1 & 1\end{array}\right]^{T}, \mathbf{s}(k)$ can be viewed as a symbolic sequence and symbolic dynamical approach is employed for the analysis of the SDMs.

In this paper, since we study the sinusoidal responses, the input signal $u(k)$ is represented in the form of $u(k)=c \sin (\Omega k+\beta)+d$ for $k \geq 0$, where $c, \Omega, \beta$ and $d$ are the amplitude, frequency, phase shift and DC offset of the input signals, respectively. Without loss of generality, we can assume that $\Omega \neq 0$. For $\Omega=0$, the input signal becomes a step signal and it is reduced to the zero or step response case, which is equivalent to the case when $c=0$ and $d$ is equal to the DC level of the input signal. Moreover, we only consider the case when the natural frequency of the loop filter $\theta$, the input signal $u(k)$ and the initial condition $\mathbf{x}(0)$ are real, that is, $\theta, c, d, \Omega, \beta \in \mathfrak{R}$ and $\mathbf{x}(0) \in \mathfrak{R}^{2}$. As a result, $y(k)$ is also real.

\section{RESULTS}

In this section, we first study the second order interpolative marginally stable bandpass SDMs with sum of numerator and denominator polynomials equal to one. Then, we will extend the results to the case when the sum of numerator and denominator polynomials not equal to one.

A. SDMs with sum of numerator and denominator polynomials equal to one

When the output of the loop filters is bounded and the output sequences are eventually periodic with period $M$ starting at the time index $k_{0}^{M}$, we can define the admissible set of eventually periodic output sequences $\Sigma \equiv \bigcup_{M \geq 1} \Sigma_{M}$ for $k \geq k_{0}$, where $\Sigma_{M}$ is the admissible set of eventually periodic output sequences with period $M$ for $k \geq k_{0}^{M}$ and $k_{0}=\max _{M \geq 1} k_{0}^{M}$, in which $M \in Z^{+} . \Sigma_{M}$ can be represented as:

$$
\Sigma_{M} \equiv\left\{\mathbf{s}_{M} \equiv\left(s\left(k_{0}^{M}\right), s\left(k_{0}^{M}+1\right), \cdots, s\left(k_{0}^{M}+M-1\right)\right): s\left(k_{0}^{M}+i\right)=s\left(k_{0}^{M}+i+p M\right) \text { for } i=0,1, \cdots, M-1 \text { and } p \in Z^{+}\right\} .
$$

Define the discrete-time Fourier coefficients of the eventually periodic output sequences with period $M$ for $k \geq k_{0}^{M}$ as $a_{p}$ for $p=0,1, \cdots, M-1$, that is $a_{p} \equiv \frac{1}{M} \sum_{k=k_{0}^{M}}^{k_{0}^{M}+M-1} s(k) e^{\frac{-j 2 \pi p k}{M}}$ for $p=0,1, \cdots, M-1$.

Then, the following lemma characterizes the constraints on $a_{p}$ for $p=0,1, \cdots, M-1$ :

\section{Lemma 1}


If $y(k)$ is bounded, the output sequences are eventually periodic with period $M$ for $k \geq k_{0}^{M}$, $|\Omega| \neq|\theta|$ and $\frac{2 \pi p}{M} \neq|\theta|$ for $p=0,1, \cdots, M-1$, then $a_{p}$ for $p=0,1, \cdots, M-1$ has to satisfy the following two constraints:

$$
\sum_{p=0}^{M-1} a_{p} a_{\bmod (q-p+M, M)}=\left\{\begin{array}{cc}
1 & q=0 \\
0 & q=1,2, \cdots, M-1
\end{array}\right.
$$

and

$$
Q\left(\sum_{p=0}^{M-1} a_{p} f_{1}(k, p)+f_{2}(k)\right)=\sum_{p=0}^{M-1} a_{p} e^{\frac{j 2 \pi p k}{M}} \text { for } k \geq k_{0}^{M}
$$

where

$$
\begin{gathered}
f_{1}(k, p)=C_{8} e^{\frac{j 2 \pi p k}{M}}+C_{9} \cos \theta k+C_{10} \sin \theta k \text { for } p=0,1, \cdots, M-1 \text { and } k \geq k_{0}^{M}, \\
f_{2}(k)=\frac{\left(x_{1}(0)-Q\left(x_{1}(0)\right)\right)(\sin (k-1) \theta-2 \cos \theta \sin k \theta)+\left(x_{2}(0)-Q\left(x_{2}(0)\right)\right)(2 \cos \theta \sin (k+1) \theta-\sin k \theta)}{\sin \theta} \\
+C_{1}+C_{2} \cos (\Omega k)+\frac{C_{3}+C_{2} \cos \Omega}{\sin \Omega} \sin (\Omega k)+C_{4} \cos (\theta k)+\frac{C_{5}+C_{4} \cos \theta}{\sin \theta} \sin (\theta k)
\end{gathered}
$$

for $k \geq 0$, and $\bmod (\alpha, M)$ represents the remainder of $\frac{\alpha}{M}$, in which

$$
\begin{gathered}
C_{1}=\frac{d(2 \cos \theta-1)}{2(1-\cos \theta)}, \\
C_{2}=\frac{\sin \Omega \operatorname{Re}\left\{C_{6}\right\}+\cos \Omega \operatorname{Im}\left\{C_{6}\right\}}{\sin \Omega}, \\
C_{3}=\frac{-\operatorname{Im}\left\{C_{6}\right\}}{\sin \Omega}, \\
C_{4}=\frac{\sin \theta \operatorname{Re}\left\{C_{7}\right\}+\cos \theta \operatorname{Im}\left\{C_{7}\right\}}{\sin \theta}, \\
C_{6}=\frac{c\left(\sin \beta+\sin (\Omega-\beta) e^{-j \Omega}\right)\left(2 \cos \theta-e^{-j \Omega}\right)}{4 \sin \left(\frac{\theta+\Omega}{2}\right) \sin \left(\frac{\theta-\Omega}{2}\right)}, \\
C_{7}=\frac{d\left(1-2 \cos \Omega+e^{-j \theta}\right)+c\left(e^{j \theta}-1\right)\left(\sin \beta+\sin (\Omega-\beta) e^{-j \theta}\right)}{4 \sin \left(\frac{\theta+\Omega}{2}\right) \sin \left(\frac{\Omega-\theta}{2}\right)\left(1-e^{-j \theta}\right)}, \\
C_{8}=\frac{e^{\frac{-j 2 \pi p}{M}}-2 \cos \theta}{4 \sin \left(\frac{\theta}{2}+\frac{\pi p}{M}\right) \sin \left(\frac{\theta}{2}-\frac{\pi p}{M}\right)},
\end{gathered}
$$




$$
\begin{aligned}
& C_{9}=\frac{1}{2 j \sin \theta}\left(\frac{e^{-j \theta}}{1-e^{\frac{j 2 \pi p}{M}} e^{j \theta}}-\frac{e^{j \theta}}{1-e^{\frac{j 2 \pi p}{M}} e^{-j \theta}}\right), \\
& C_{10}=\frac{1}{2 j \sin \theta}\left(\frac{e^{-j\left(\frac{\pi}{2}-\theta\right)}}{1-e^{\frac{j 2 \pi p}{M}} e^{-j \theta}}-\frac{e^{j\left(\frac{\pi}{2}-\theta\right)}}{1-e^{\frac{j 2 \pi p}{M}} e^{j \theta}}\right),
\end{aligned}
$$

$\operatorname{Re}\{\alpha\}$ and $\operatorname{Im}\{\alpha\}$ denote, respectively, the real and imaginary part of $\alpha$. Proof:

Since $y(k)$ is bounded and the output sequences are eventually periodic with period $M$ for $k \geq k_{0}^{M}$, the Fourier representation of the eventually periodic output sequences with period $M$ for $k \geq k_{0}^{M}$ exists. Since $(Q(y(k)))^{2}=1$ for $k \geq 0$, equation (7) has to be satisfied. Besides, since $|\Omega| \neq|\theta|$ and $\frac{2 \pi p}{M} \neq|\theta|$ for $p=0,1, \cdots, M-1$, then by computing the outputs of the loop filter due to, respectively, the zero input and without quantizer feedback, the zero initial condition and without quantizer feedback, and zero initial condition and zero input, equation (8) has to be satisfied, where the coefficients in equation (8) are obtained by grouping the terms correspondingly. Hence, this completes the proof.

The importance of Lemma 1 is that it provides information to check whether a binary eventually periodic sequence with period $M$ for $k \geq k_{0}^{M}$ is an eventually periodic admissible sequence generating a bounded trajectory for the SDMs or not. For the absolute value of the input sinusoidal frequency and that of the harmonics of the eventually periodic output sequence with period $M$ for $k \geq k_{0}^{M}$ not equal to that of the natural frequency of the loop filter, if we cannot find an initial condition $\mathbf{x}(0)$ and a starting time index $k_{0}^{M}$ such that equations (7) and (8) are satisfied, then the test sequence is not an eventually periodic admissible sequence generating a bounded trajectory for the SDMs.

Since the input signals are periodic, the output of the loop filter and the output sequences are assumed to be bounded and eventually periodic with period $M$ for $k \geq k_{0}^{M}$, respectively, the spectrum of $y(k)$ will consist of impulses. As a result, fixed points and limit cycles may occur but fractal and irregular chaotic patterns would not exhibit on the phase portrait. Figure 1 shows some phase portraits of these SDMs. Although the output sequences are eventually periodic with period $M$ for $k \geq k_{0}^{M}$, there are many different patterns exhibiting on the phase portraits as shown in Figure 1.

In Lemma 1, we assume that both the absolute value of the input sinusoidal frequency and that of 
the harmonics of the eventually periodic output sequences with period $M$ for $k \geq k_{0}^{M}$ are not equal to the absolute value of the natural frequency of the loop filter. However, as the bandpass SDMs shape away the noise from the natural frequency of the loop filter, the input sinusoidal frequency is usually equal to the natural frequency of the loop filter. This case will be discussed in the following lemma.

\section{Lemma 2}

Suppose $y(k)$ is bounded and the output sequences are eventually periodic with period $M$ for $k \geq k_{0}^{M}$. Let $q$ be a positive rational number. $|\Omega|=|\theta|=q \pi$ if and only if $\exists p^{*} \in\{0,1, \cdots, M-1\}$ such that $p^{*}=\frac{M|\theta|}{2 \pi}$ and $a_{p^{*}}=\frac{c}{2} e^{-j\left(\frac{\pi}{2}-\beta\right)}$.

Proof:

Since the output sequences are eventually periodic with period $M$ for $k \geq k_{0}^{M}$ and $y(k)$ is bounded, the Fourier representation on the eventually periodic output sequences with period $M$ for $k \geq k_{0}^{M}$ exists. If $\exists p^{*} \in\{0,1, \cdots, M-1\}$ such that $p^{*}=\frac{M|\theta|}{2 \pi}$, then there exists an impulse located at the natural frequency of the loop filter on the spectrum of the output sequences. This sinusoidal component may cause resonance effect and has to be cancelled by the input sinusoidal signals because $y(k)$ is bounded. Hence, the input signals have to contain a sinusoidal component located at the natural frequency of the loop filter and this proves that $|\Omega|=|\theta|$. To prove $|\theta|$ is a rational multiple of $\pi$, since $p^{*}=\frac{M|\theta|}{2 \pi}$ and $p^{*}$ is an integer, the result follows directly. For the converse, when $|\Omega|=|\theta|$, in order to cancel the resonance effect generated by the input sinusoidal component, the Fourier component of the output sequences should have a component located at the natural frequency of the loop filter with the value $a_{p^{*}}=\frac{c}{2} e^{-j\left(\frac{\pi}{2}-\beta\right)}$. This proves the converse and completes the whole proof.

The importance of Lemma 2 is for the proof of Theorem 1 which is stated below. From Lemma 2, we see that, unlike linear systems, the trajectory of the nonlinear SDMs does not necessarily diverge even though the input sinusoidal frequency is equal to the natural frequency of the loop filter.

Figure 2b, Figure 2d and Figure 2a show, respectively, the frequency spectrum of $x_{1}(k)$, the frequency spectrum of $y(k)$ and the phase portrait of the SDMs when $u(k)=0.05 \sin \left(\frac{\pi k}{2}\right)$ for $k \geq 0$, $\theta=\frac{\pi}{2}$ and $\mathbf{x}(0)=\left[\begin{array}{ll}0.1 & 0.2\end{array}\right]^{T}$, in which the red and black lines on Figure 2b, Figure 2c and Figure 2d represent, respectively, the frequency spectrum of the corresponding spectra and the magnitude response due to the initial condition of the loop filter. It can be seen from Figure 2a that the trajectory 
is bounded. Figure 2c shows the frequency spectrum of $s_{1}(k)$. It can be seen from Figure 2c that $s_{1}(k)$ is eventually periodic and the SDM exhibits limit cycle behavior. Since $\theta=\frac{\pi}{2}$, according to Lemma 2, we can conclude that there exists an impulse located at the natural frequency of the loop filter on the spectrum of eventually periodic output sequences, which is also the input sinusoidal frequency, and the corresponding Fourier coefficient is $\frac{c}{2} e^{-j\left(\frac{\pi}{2}-\beta\right)}$, as shown in Figure 2c.

Now we extend Lemma 2 to the cases when the output sequences are aperiodic.

\section{Theorem 1}

If $y(k)$ is bounded, then $|\Omega|=|\theta|$ if and only if there exists an impulse located at the natural frequency of the loop filter on the spectrum of output sequences. Furthermore, for bounded $y(k)$ and $|\Omega|=|\theta|, s(k)$ is aperiodic if and only if $\theta$ is not a rational multiple of $\pi$.

\section{Proof:}

The proof is similar to that of Lemma 2, so it is omitted here.

The importance of this theorem is to provide information to check whether there is an impulse located at the natural frequency of the loop filter on the spectrum of the output sequences or not. When the output of the loop filter is bounded, the existence of an impulse located at the natural frequency of the loop filter on the spectrum of the output sequences of the SDMs is equivalent to the fact that the absolute value of the input sinusoidal frequency is equal to that of the natural frequency of the loop filter. Moreover, if $y(k)$ is bounded and $|\Omega|=|\theta|$, then the occurrence of limit cycles is equivalent to the fact that the absolute value of the input sinusoidal frequency or that of the natural frequency of the loop filter is a rational multiple of $\pi$. Based on these two results, it suggests that the absolute value of the input sinusoidal frequency, as well as that of the natural frequency of the loop filter, should not be set at a rational multiple of $\pi$ for avoiding the occurrence of limit cycles.

If $y(k)$ is bounded, $|\Omega|=|\theta|$ and $\theta$ is not a rational multiple of $\pi$, then fractal or irregular chaotic patterns may be exhibited on the phase portrait. Figure 3b, Figure 3d and Figure 3a show, respectively, the frequency spectrum of $x_{1}(k)$, the frequency spectrum of $y(k)$ and the phase portrait of the SDM when $u(k)=0.1 \sin (1.5 k)$ for $k \geq 0, \theta=1.5$ and $\mathbf{x}(0)=\left[\begin{array}{ll}0.1253 & 0.2877\end{array}\right]^{T}$, in which the red and black lines on Figure 3b, Figure 3c and Figure 3d represent, respectively, the frequency spectrum of the corresponding spectra and the magnitude response due to the initial condition of the loop filter. It can be seen from Figure 3a that the trajectory is bounded. According to Theorem 1, since $|\Omega|=|\theta|$, we can conclude that there exists an impulse located at the natural frequency of the loop filter on the spectrum of output sequences, which is also the input sinusoidal frequency, as shown in Figure 3c. In this example, since the natural frequency of the loop filter is 1.5 , which is not a rational 
multiple of $\pi$, so according to Theorem $1, s(k)$ is aperiodic. Figure 3c shows the frequency spectrum of $s_{1}(k)$. It can be seen from Figure $3 c$ that $s_{1}(k)$ is aperiodic and the SDM exhibits chaotic behavior, as predicted by the theorem. Figure 4 shows another example when $u(k)=0.01 \sin (-1.746 k)$ for $k \geq 0, \theta=-1.8416$ and $\mathbf{x}(0)=\left[\begin{array}{ll}0.01 & 0.01\end{array}\right]^{T}$. In this case, the trajectory is still bounded. Since $|\Omega| \neq|\theta|$, so according to Theorem 1 , there does not exist an impulse located at the natural frequency of the loop filter on the spectrum of output sequences, as shown in Figure 4c.

Next, we will explore the conditions when $y(k)$ is unbounded.

\section{Lemma 3}

Suppose that the output sequences are eventually periodic with period $M$ for $k \geq k_{0}^{M}$ and $|\Omega|=|\theta|=q \pi$, where $q$ is a positive rational number. If $\forall p^{*} \in\{0,1, \cdots, M-1\}, a_{p^{*}} \neq \frac{c}{2} e^{-j\left(\frac{\pi}{2}-\beta\right)}$, then $|y(k)| \rightarrow+\infty$ for $k \rightarrow+\infty$.

\section{Proof:}

The proof follows directly from Lemma 2, so it is omitted here.

The importance of Lemma 3 is to provide information to check whether the output of the loop filter diverges or not. It is worth noting that even though the output sequences are eventually periodic with period $M$ for $k \geq k_{0}^{M}$, the resonance effect introduced by the input signals may not be canceled by that of the eventually periodic output sequences when the corresponding Fourier coefficient of the eventually periodic output sequences is not equal to $\frac{c}{2} e^{-j\left(\frac{\pi}{2}-\beta\right)}$. If this is the case, then the output of the loop filter will diverge. Compared to the results reported in [5] that the second order interpolative bandpass SDMs are globally stable for both zero and step inputs no matter where the initial conditions are, the global stability for the sinusoidal response cases is not guaranteed.

Figure 5a, Figure 5c, Figure 5e and Figure 5b show, respectively, the response of $x_{1}(k)$, the frequency spectrum of $x_{1}(k)$, the frequency spectrum of $y(k)$ and the phase portrait of the SDM when $u(k)=10 \sin \left(\frac{\pi k}{10}\right)$ for $k \geq 0, \theta=\frac{\pi}{10}$ and $\mathbf{x}(0)=\left[\begin{array}{ll}0.3273 & 0.1746\end{array}\right]^{T}$, in which the red and black lines on Figure 5c, Figure 5d and Figure 5e represent, respectively, the frequency spectrum of the corresponding spectra and the magnitude response due to the initial condition of the loop filter. Figure $5 d$ shows the frequency spectrum of $s_{1}(k)$. It can be seen from Figure $5 d$ that $s_{1}(k)$ is eventually periodic and the SDM exhibits limit cycle behavior with period 20. According to Lemma 3, since $|\Omega|=|\theta|=q \pi$ where $q$ is a positive rational number, and $\forall p^{*} \in\{0,1, \cdots, M-1\} \quad a_{p^{*}} \neq \frac{c}{2} e^{-j\left(\frac{\pi}{2}-\beta\right)}$, we can conclude that the trajectory is unbounded, as shown in Figure 5a and Figure 5b.

\section{B. SDMs with sum of numerator and denominator polynomials not equal to one}


Now we extend the results to the case when the sum of the numerator and denominator polynomials of the loop filter is not equal to one. That is, by denoting the loop filter of a second order interpolative marginally stable bandpass SDMs as $F^{\prime}(z)=\frac{\gamma z^{-1}+\kappa z^{-2}}{1-2 \cos \theta z^{-1}+z^{-2}}$, where $\gamma$ and $\kappa$ are real and $(\gamma, \kappa) \neq(-2 \cos \theta, 1)$.

\section{Theorem 2}

If $\kappa>0$, then $\exists \mathbf{x}(0) \in \mathfrak{R}^{2}$ such that $|y(k)| \rightarrow+\infty$ for $k \rightarrow+\infty$.

\section{Proof:}

The proof is shown in [8].

The importance of this theorem is to provide information to check whether the output of the loop filter diverges or not. If $\kappa>0$, then the output of the loop filter may be unstable. Hence, it is important to choose the numerator coefficients such that $\kappa \leq 0$.

\section{High order interpolative marginally stable bandpass SDMs}

Although there are limits on the applications of second order interpolative marginally stable bandpass SDMs, high order ones are found many applications and part of the results in Theorem 1 is applied for those high order ones. If the absolute value of the input sinusoidal frequency is not equal to that of the natural frequencies of the loop filter and there is no impulse located at the natural frequencies of the loop filter on the spectrum of the output sequences, then the output of the loop filter is bounded. Figure 6 shows responses of 8 order interpolative marginally stable bandpass SDMs designed via the Matlab sigma-delta toolbox [9] with oversampling ratio 64 and center frequency $\frac{\pi}{2}$. It can be checked that the natural frequencies of the loop filter are $e^{ \pm j 1.5497}, e^{ \pm j 1.5625}, e^{ \pm j 1.5791}$ and $e^{ \pm j 1.5919}$. Assume that the input of the SDM is $u(k)=0.1 \sin \left(\frac{\pi k}{2}\right)$ for $k \geq 0$. Then the absolute value of the input sinusoidal frequency is not equal to that of the natural frequencies of the loop filter. Figure 6b, Figure $6 \mathrm{~d}$ and Figure 6a show, respectively, the frequency spectrum of $x_{1}(k)$, the frequency spectrum of $y(k)$ and the plot of $x_{2}(k)$ against $x_{1}(k)$ under zero initial condition, in which the red and black lines on Figure 6b, Figure 6c and Figure 6d represent, respectively, the frequency spectrum of the corresponding spectra and the natural frequencies of the loop filter. It can be seen from Figure $6 \mathrm{c}$ that there is no impulse located at the natural frequencies of the loop filter on the spectrum of the output sequences, so the trajectory is bounded. It can be seen from Figure 6a that $x_{1}(k)$ is bounded and the trajectory is confirmed with certain region in the state space.

\section{CONCLUSION}

Most of the existing stability analysis of SDMs is restricted to time domain analysis and step responses for lowpass systems. In this work, we have used frequency domain analysis to investigate the stability of bandpass SDMs with respect to sinusoidal responses. The main contribution of this paper is to study the admissibility conditions on the Fourier coefficients of the eventually periodic output sequences that generate bounded trajectories, and to analyze the stability of sinusoidal 
responses of second order interpolative bandpass SDMs in the frequency domain. In Lemma 1, we provided conditions as to whether an eventually periodic output sequence is an admissible sequence for generating a bounded trajectory. From Lemma 2 we showed that the trajectory of an SDM does not necessarily diverge even though the input sinusoidal frequency is equal to the natural frequency of the loop filter. Theorem 1 then generalizes Lemma 2 to the aperiodic case and provides information for the occurrence of fractal and chaotic behaviors. Finally, Lemma 3 provided conditions for the divergent behavior. These theoretical results were confirmed by simulation on a variety of SDMs with different sinusoidal responses and can be further extended to high order interpolative bandpass SDMs. High order bandpass SDMs are useful in many practical systems because the oversampling ratios of bandpass SDMs are usually lower than that of lowpass SDMs. By applying the developed theory in this paper, the stability of these bandpass SDMs can be checked easily.

\section{ACKNOWLEDGEMENT}

The work obtained in this paper was supported by a research grant from Queen Mary, University of London.

\section{REFERENCES}

[1]James C. Candy, “A use of limit cycle oscillations to obtain robust analog-to-digital converters,” IEEE Transactions on Communications, vol. COM-22, no. 3, pp. 298-305, 1974.

[2]Orla Feely, “A tutorial introduction to non-linear dynamics and chaos and their application to sigma-delta modulators,” International Journal of Circuit Theory and Applications, vol. 25, no. 5, pp. 347-367, 1997.

[3]Georgi P. Petkov and Anthony C. Davies, "Constraints on constant-input oscillations of a bandpass sigma-delta modulator structure,” International Journal of Circuit Theory and Applications, vol. 25, no. 5, pp. 393-405, 1997.

[4]Anthony C. Davies and Georgi P. Petkov, "Zero-input oscillation bounds in a bandpass $\Sigma \Delta$ modulator,” Electronics Letters, vol. 33, no. 1, pp. 28-29, 1997.

[5]Peter Ashwin, Xin-Chu Fu and Jonathan Deane, "Properties of the invariant disk packing in a model bandpass sigma-delta modulator,” International Journal of Bifurcations and Chaos, vol. 13, no. 3, pp. 631-641, 2003.

[6]Tsung-Yuan Chang and Steven B. Bibyk, "Quantization error analysis of second order bandpass delta-sigma modulator with sinusoidal inputs,” Analog Integrated Circuits and Signal Processing, vol. 26, no. 3, pp. 213-228, 2001.

[7]Sundeep Rangan and Bosco Leung, “Quantization noise spectrum of double-loop sigma-delta converter with sinusoidal input,” IEEE Transactions on Circuits and Systems-II: Analog and Digital Signal Processing, vol. 41, no. 2, pp. 168-173, 1994.

[8]Charlotte Yuk-Fan Ho, Bingo Wing-Kuen Ling and Joshua D. Reiss, "Design of second order interpolative sigma delta modulators with guarantee global stability," submitted to IEEE Transactions Circuits and Systems-I: Regular Papers.

[9]Richard Schreier, The delta-sigma modulators toolbox version 6.0, Analog Devices Inc., $1^{\text {st }}$ Jan 
2003. 

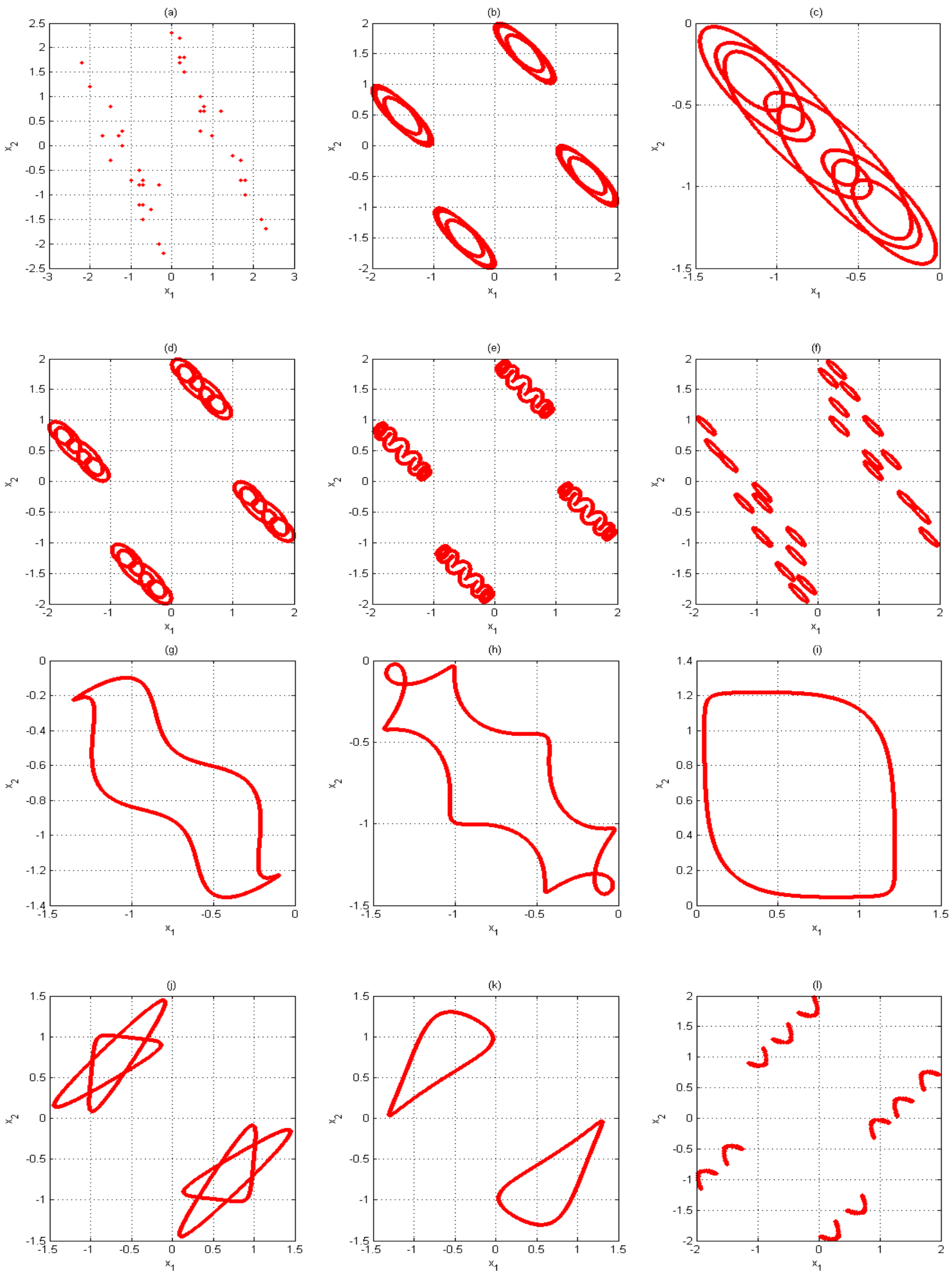

Figure 1: Phase portraits of second order interpolative bandpass SDMs when the output sequences are eventually periodic with period $M$ for $k \geq k_{0}^{M}$. 

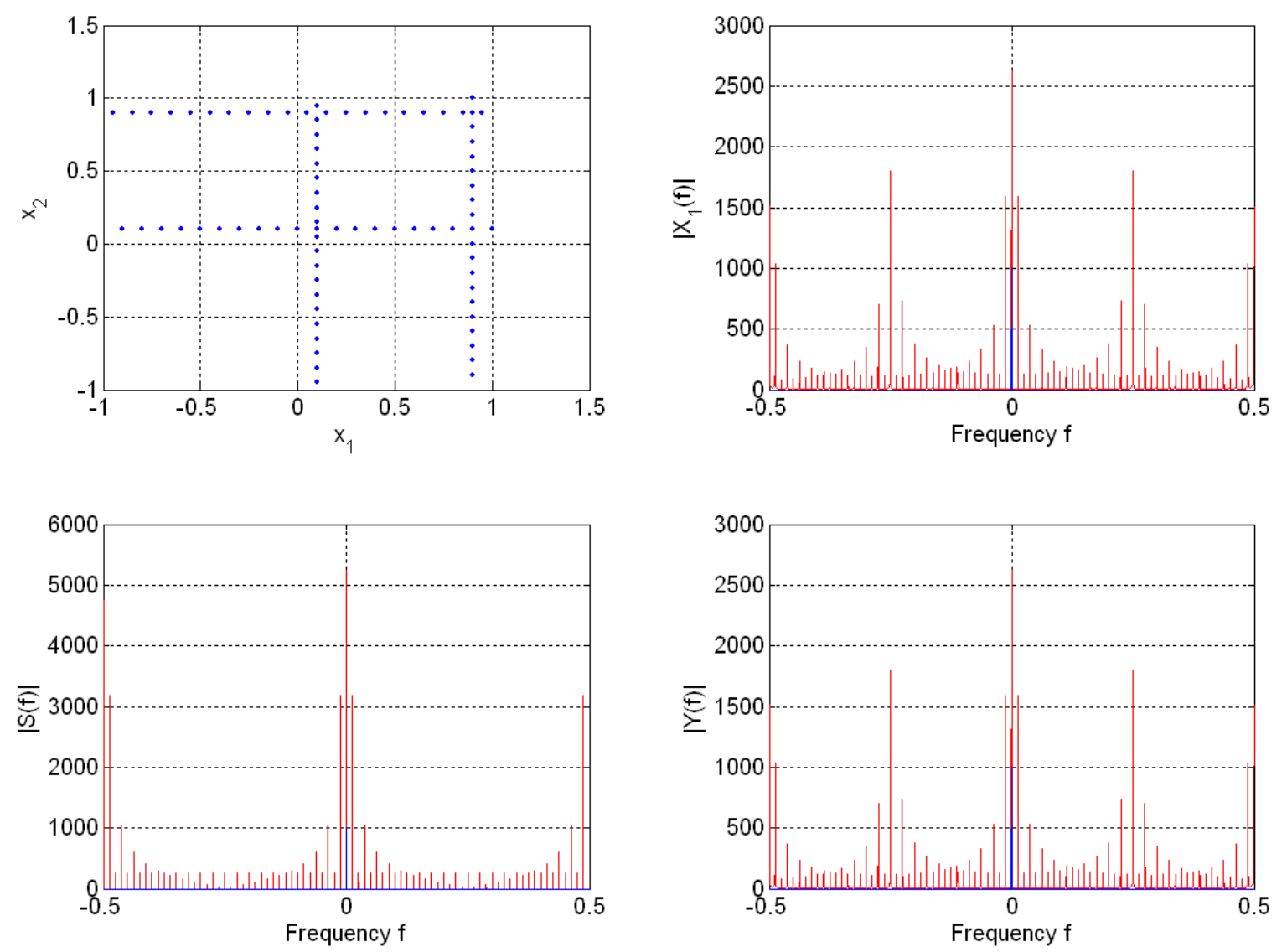

Figure 2: (a) Phase portrait of the second order interpolative bandpass SDM. (b) Frequency spectrum of $x_{1}(k)$. (c) Frequency spectrum of $s_{1}(k)$. (d) Frequency spectrum of $y(k)$.
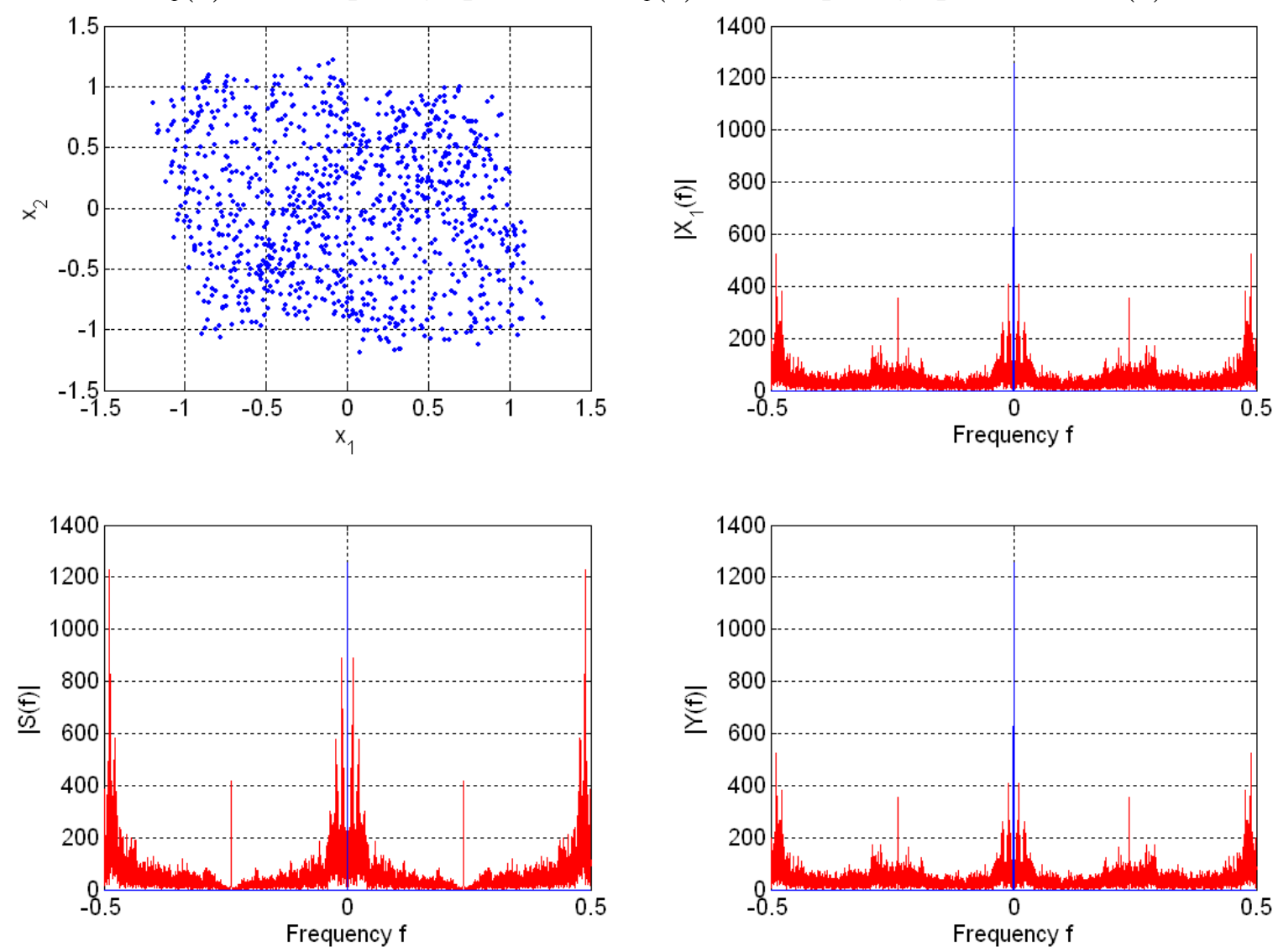

Figure 3: (a) Phase portrait of the second order interpolative bandpass SDM. (b) Frequency spectrum of $x_{1}(k)$. (c) Frequency spectrum of $s_{1}(k)$. (d) Frequency spectrum of $y(k)$. 

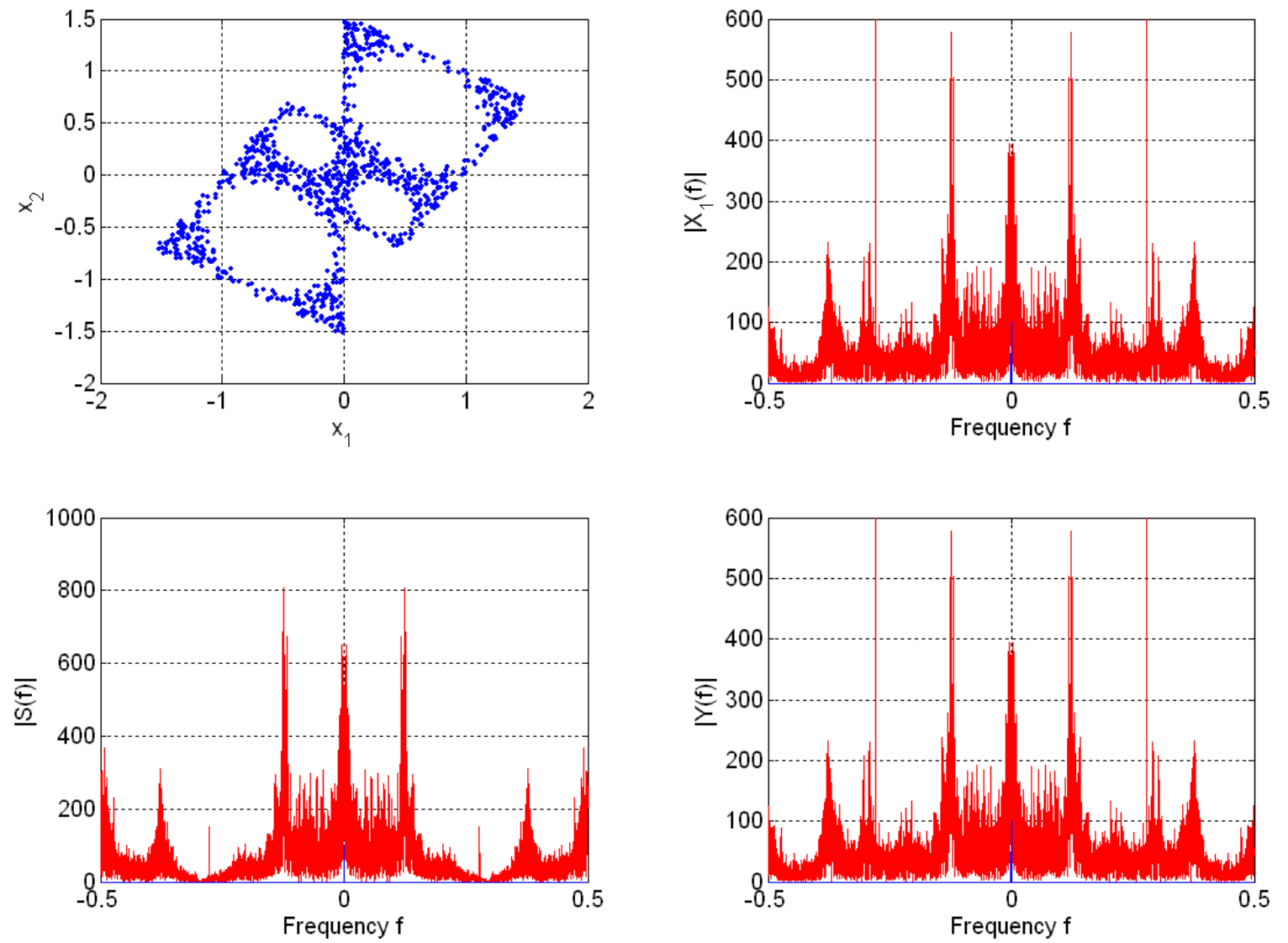

Figure 4: (a) Phase portrait of the second order interpolative bandpass SDM. (b) Frequency spectrum of $x_{1}(k)$. (c) Frequency spectrum of $s_{1}(k)$. (d) Frequency spectrum of $y(k)$.
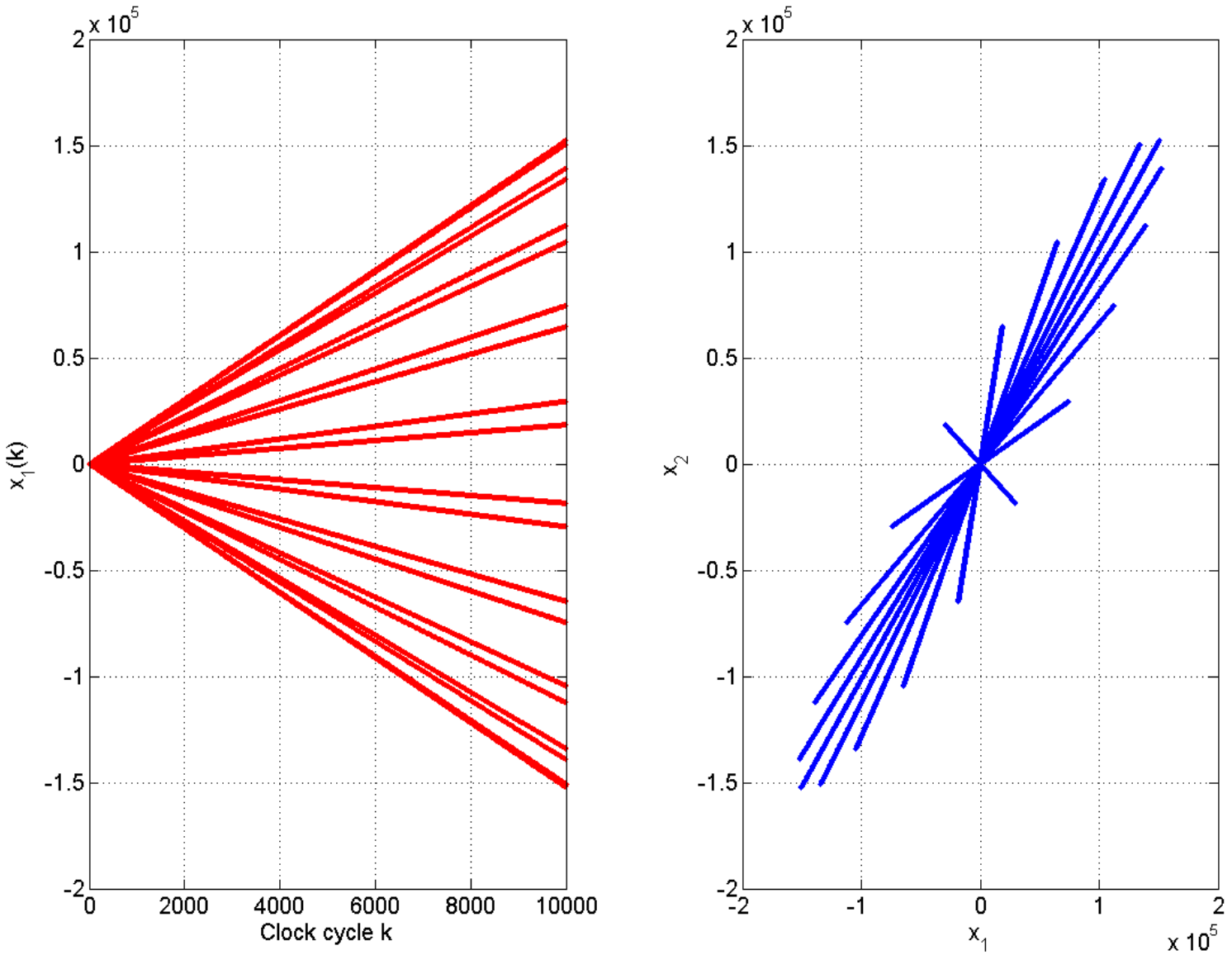

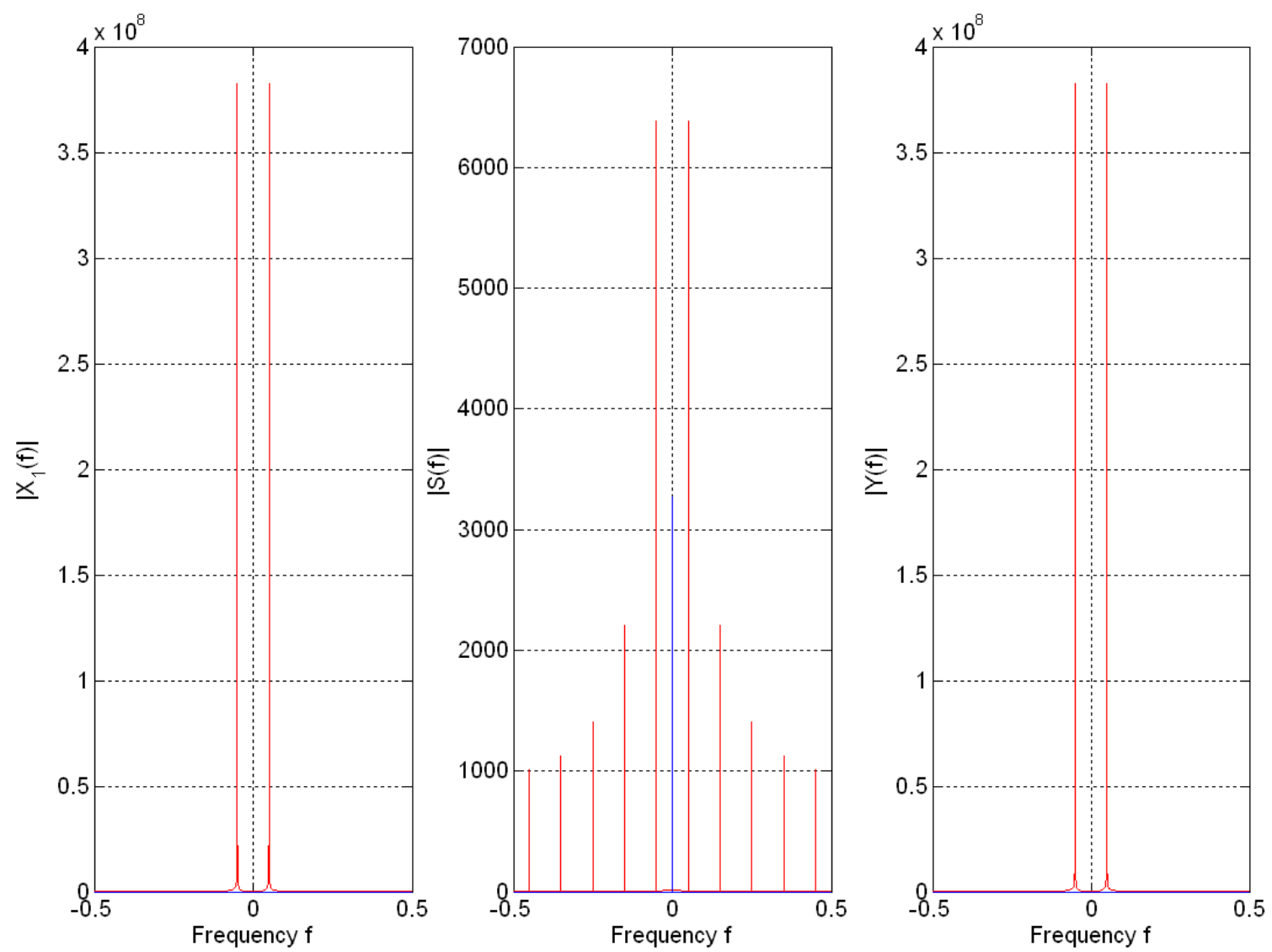

Figure 5: (a) Response of $x_{1}(k)$. (b) Phase portrait of the second order interpolative bandpass SDM. (c) Frequency spectrum of $x_{1}(k)$. (d) Frequency spectrum of $s_{1}(k)$. (e) Frequency spectrum of $y(k)$.
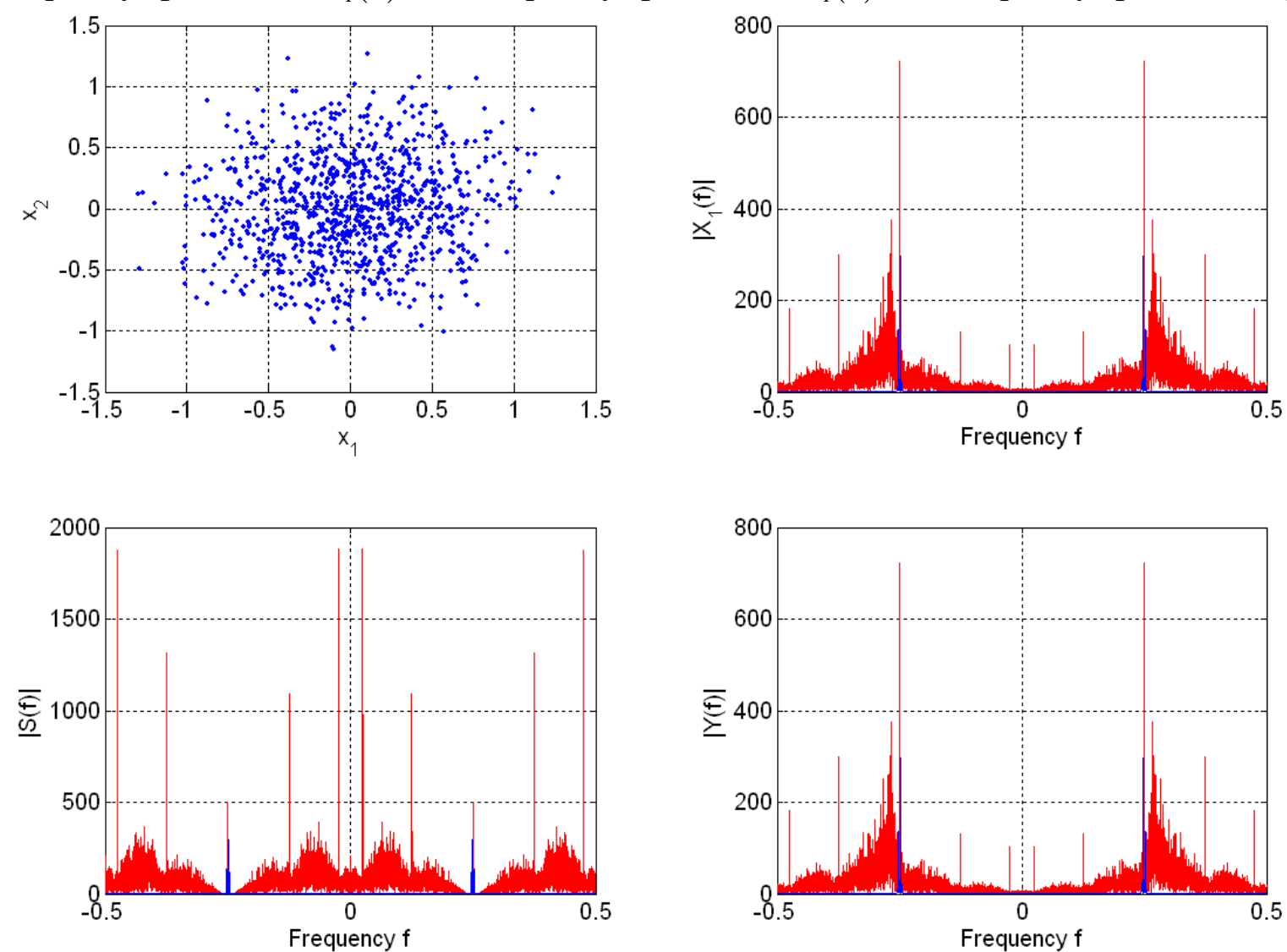

Figure 6: (a) Plot of $x_{2}(k)$ against $x_{1}(k)$. (b) Frequency spectrum of $x_{1}(k)$. (c) Frequency spectrum of $s_{1}(k)$. (d) Frequency spectrum of $y(k)$. 